\title{
Back to the Future: What Have We Failed to Learn? How Does the Future Look?
}

\section{Marion Ball}

IMIA Award Presentation MEDINF0, Cape Town, September 2010

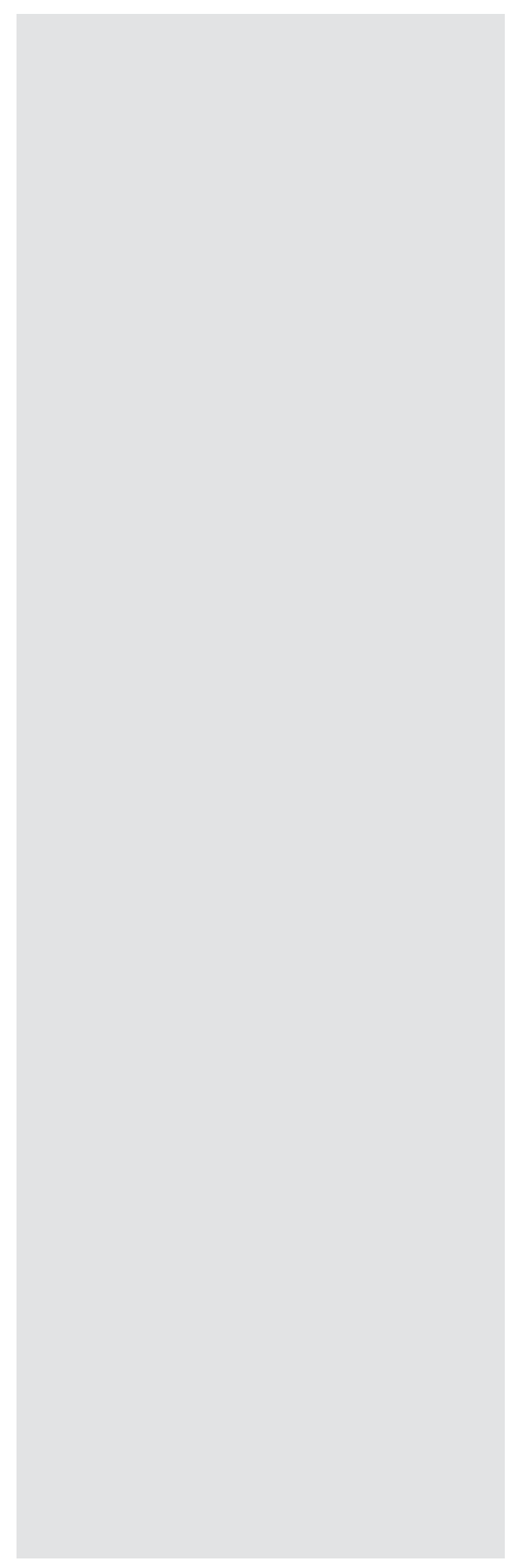

Marion Ball is Past President of the International Medical Informatics Association (IMIA), presently Senior Advisor to the Healthcare and Life Sciences Institute, IBM Research, Professor Emerita, Johns Hopkins University, Member, Institute of Medicine-National Academy of Sciences, USA, Member of the Board Of Regents of the National Library of Medicine, Fellow of the American College of Medical Informatics (ACMI), Past Board member and Fellow of the Health Information Management and Systems Society (HIMSS), American Health Information Management Association (AHIMA) Medical Library Association (MLA) and the College of Health Information Management Executives (CHIME), American Academy of Nursing (FAAN).

\section{Framing the Problem}

In 1948, Claude E. Shannon published the paper "A Mathematical Theory of Communication" [1], founding the field of Information Theory and establishing the basis for coding and transmitting information over communication channels.

His work, together with that of Norbert Weiner on control theory, provided the basis for much of modern communication and control, or cybernetics, which, coupled with the development of computers in the decade of the 1950 's, led to dramatic progress in automation of all types of industrial processes. Claude Shannon carried out a number of experiments with a sys- tem called Theseus, trying to solve a maze which can be thought of as a precursor of work on artificial intelligence.

In medicine, progress steadily accelerated through the next two decades, leading to the development of the first hospital and clinical information systems. I was involved in organizing the First IMIA Working Conference on Hospital Information Systems, which was held here, in Cape Town, South Africa, in 1979. The proceedings were published as a book [2] (Figure 1).

The main themes of this IMIA Working Conference, expressed in more current terms, were:

- Human Factors

- It is the Patient who is at the Center

- Clinical User Support (the point of Care)

- Real-time education (at the point of care)

- Clinical User Performance

The contrast to today's situation is informative. There has been, especially over the past decade, a major shift in public policy leading to the rapid expansion of health informatics, eHealth, and other ways of describing Health Information Technology (HIT). This has worked well for clinical applications which are routine, and standardized, such as those which involve laboratory and image data, but for primary care or internal medicine or other more complex clinical environments, the introduction of computer technology has often added to the clinician's time and work. A major challenge of HIT, then, is to develop systems that actually save time, and simplify work, rather than the opposite. 


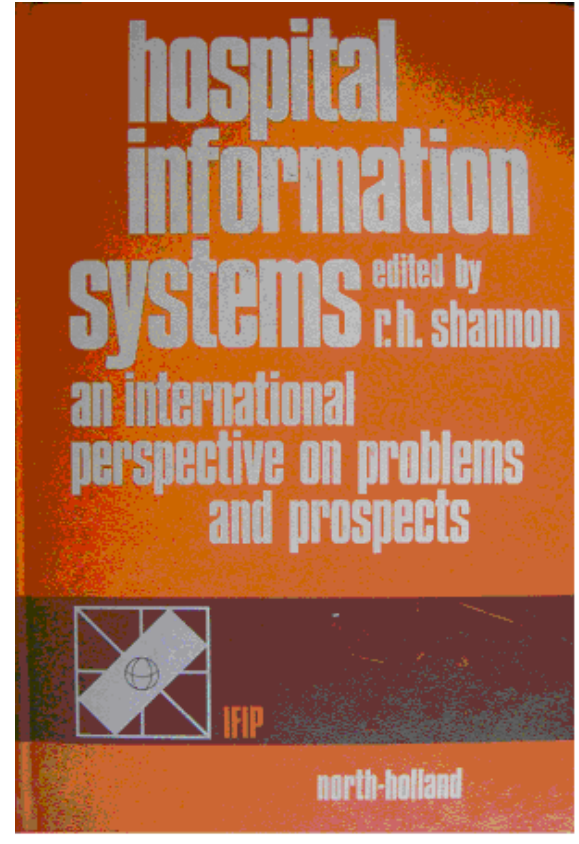

Fig. 1 The Proceedings of the First IMIA Working Conference on Hospital Information Systems, 1979.

Another great challenge involves the role of HIT in patient safety. The number of patients who die due to medical mistakes has been estimated to range from 98,000 to 195,000 , with more than 57,000 dying from inadequate care. Over the 2003 to 2005 period it was reported that hospital incidents increased by $3 \%$ [3], and now, a new type of medical error frequently arises due to the involvement of computer systems. In Intensive Care Units (ICUs) the total number of preventable deaths was 166,798 , with the breakdown by various causes, as reported by Pronovost et al [4] given in Table 1.

Another major challenge in medical informatics arises from the failure to communicate, as for example, in efforts to try to reduce the risk of stroke.

The percentage of patients who do this by using the drug Pravachol is $19 \%$, and they incur a cost of $\$ 4.84$ for one $40 \mathrm{mg}$ tablet $3 / 15 / 07$ at Walgreen's online.

In contrast, the simple dietary change of just eating fish once a week can reduce the risk of stroke by $22 \%$. Controlling high blood pressure reduces risk by $35-45 \%$ (often with diuretics that
Table 1 Percentage of Patients not Receiving Care from various Potential Interventions that could Prevent Death in the ICU (from [4])

\begin{tabular}{|l|c|c|}
\hline Care process & $\begin{array}{c}\% \text { Not } \\
\text { Receiving }\end{array}$ & $\begin{array}{c}\text { Preventable } \\
\text { Deaths }\end{array}$ \\
\hline ICU Physician & $77 \%$ & 134,640 \\
Sepsis Drug & $89 \%$ & 10,311 \\
Steroids in Sepsis & $50 \%$ & 9,500 \\
Glucose Control & $75 \%$ & 12,347 \\
\hline
\end{tabular}

cost less than $\$ 0.15 /$ day), while moderate exercise for less than 2 hours/week in the elderly reduces it considerably by about $60 \%$ [4]. The effect of exercise and diet on health was well illustrated in a cartoon from The Economist in its December 2003 issue, parodying evolutionary development, which ended with an obese modern man holding a soft-drink.

The fragmentation of medical care and the intrusion of administrative and bureaucratic managers to deal with billing, insurance, utilization processes, and other factors has been widely commented, contributing to the unsustainable growth in costs of healthcare. One can only quote from Einstein that: "Insanity: Continuing to do the same thing and expecting different results."

These problems can be addressed reengineering health care systems, while recognizing that not all change requires IT for success and that health IT needs to capitalize on innovations by developing intelligent software tools that can help reduce, rather than increase costs.

\section{Reengineering for Patient- Centered Care}

The designers of healthcare software systems could learn from other industries, where success is achieved by focusing on the necessary efficiencies and performance simultaneously. A not-soobvious example that was used to improve hospital efficiency comes from car racing's Formula 1, where in 7 seconds a 20-member crew switches a car's tires, adjusts its front wing, and cleans the air vents - all based on studies conducted by human-factors experts to study how pit crews perform, uncovering small errors that could cause important delays [6]. The contrast between the Formula 1 Ferrari team and the hospital studied involved

a) training for the worst contingencies vs. dealing with problems "just as they came up"; b) having each member of the pit team with very specific assigned tasks, carrying out their jobs in a specific sequence and in silence vs. having no specific sequence of connecting or disconnecting to machines, and chaotic communication among team members; c) having a clear person in charge who signals when and how the car enters and leaves the pit vs. having no clear person in charge. The results of applying this approach in a hospital setting was that technical errors per handover were reduced by $42 \%$, "information handover omissions" were down $49 \%$, and there was slightly less time to execute each handover.

A large part of the clinical workforce that must make IT work in the clinic is in nursing, and it is here that the process of Technology Informatics Guiding Education (TIGER) [7] developed by the Alliance for Nursing Informatics (ANI), sponsored by the major nursing organizations in the USA and the American Medical Informatics Association (AMIA), becomes an important driver for change. How to make health information technology (HIT) work effectively and efficiently for clinicians (so it subtracts work) is therefore HIT's greatest challenge.

To quote Einstein again, "No problem can be solved within the same consciousness which caused it.", which, when combined with Claude Shannon's insight that "Information is a message that reduces uncertainty" can help us overcome the deluge of clinical data that is the daily challenge of clinical informatics. 
198

Ball

Re-engineering or transforming health care processes to implement "smart point of care solutions" involve the following main requirements:

- Anticipate needs - have data/information ready before clinician needs it

- Understand the individual clinician's work-flow

- Context is everything, but so is speed

- Hide complexity with simplicity ('magical' IT)

- Reduce the amount of work!

The move from overwhelming amounts of healthcare data, innovations that can help in harnessing clinical data is well exemplified in Project Artemis at the Toronto Sick Children's Hospital. Using IBM's InfoSphere Streams system it was configured to perform online analytics on streaming data coming from medical instruments used to monitor premature infants, giving up to 24 hour 'advanced warning' of the onset of nosocomial infection [8].

Another important set of insights comes from studies on the amount of time involved in nursing workflow, which involve 2-4 miles, or $20 \%$ of time of walking, $10 \%$ to $15 \%$ of RN time doing "non-RN" work, and only $30 \%$ $40 \%$ in direct patient care. The Smart Room concept developed by the University of Pittsburgh Medical Center (UPMC) in a partnership with IBM [9], when applied to a nursing station resulted in a $57 \%$ decrease in charting time, $69 \%$ reduction of time in documenting daily living tasks, and an $82 \%$ reduction in time to document vital signs.

To conclude this presentation, we can think about the latest dramatic developments in using informatics methods in pharmacogenomics, which are leading to the personalization, or patient-tailored medicine, by recalling Osler's dictum:
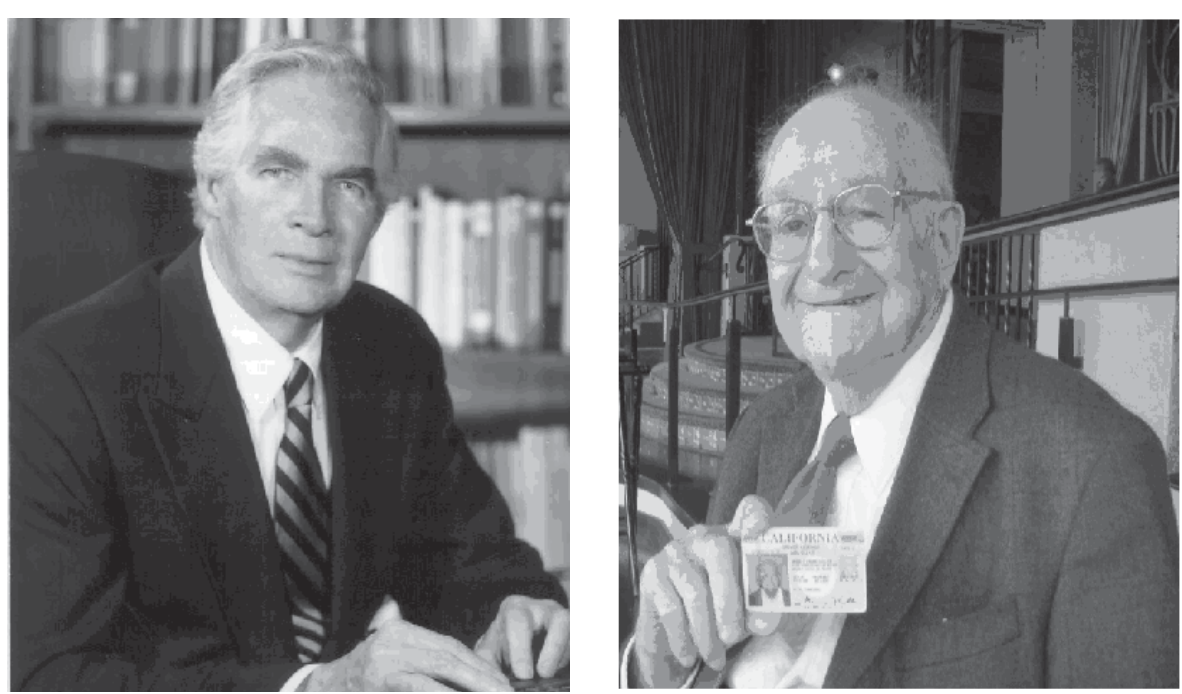

Fig. 2 Drs. Donald A.B. Lindberg and Morris F. Collen

"It is much more important to know what sort of patient has a disease than what sort of disease a patient has." [5]. This leads me to suggest the following major challenges for IMIA:

1. Relating genomics, proteomics, and metabolomics to "what sort of patient has a disease"

2. Using 'omics-based health' at the point of care, smartly

3. Delivering predictable and optimized health for all citizens of our countries.

I would like to dedicate this keynote to my two mentors over many many years: Drs Morrie Collen and Don Lindberg (Figure 2). (Dr. Donald A. B. Lindberg is the director of the National Library of Medicine, and Dr. Morris Collen, Consultant and Director Emeritus, Permanente Medical Group.)

Don has been one of the reasons IMIA exists and asked me to extend his best wishes for a successful meeting. Here you see Morrie holding his latest drives license valid to his 100th birthday!! He plans to drive us to his 100th birthday party!

\section{References}

1. Shannon CE. A Mathematical Theory of Communication. Bell System Technical Journal 1948;27:379-423; 623-56.

2. Shannon RH, editor. Hospital Information Systems: An International Perspective on Problems and Prospects. Amsterdam: North Holland; 1979.

3. HealthGrades, Inc. Fourth Annual Patient Safety in American Hospitals Study, September 2007.

4. Pronovost PJ, Rinke ML, Emery K, Dennison $\mathrm{C}$, Blackledge C, Berenholtz SM. Interventions to reduce mortality among patients treated in intensive care units. J Crit Care 2004; 13(3):158-64.

5. Abramson J. Overdosed America: The Broken Promise of American Medicine. New York: Harper Collins; 2004.

6. Naik G. New formula: A hospital races to learn lessons of Ferrari pit stop. Wall Street Journal, November 14, 2006.

7. Gugerty B, Delaney C. TIGER Informatics Competencies Collaborative (TICC) Final Report, August 2009. Available from tigercompetencies. pbworks.com/f/TICC_Final.pdf

8. James A. The Artemis Project: Harnessing high volume, high fidelity physiological data streams for intensive care decision support. Available from https://www.950.ibm.com/events/wwe/ca/canada/ Andrew_James.pdf

9. www.pittsburghlive.com/x/pittsburghtrib/ lifestyles/ 